\title{
High TSC22D3 and low GBP1 expression in the liver is a risk factor for early recurrence of hepatocellular carcinoma
}

\author{
KEIKO TAKAGI $^{1}$, TADATOSHI TAKAYAMA ${ }^{1}$, HIROKI NAGASE ${ }^{2}$, MASAMICHI MORIGUCHI $^{1}$, XIAOFEI WANG ${ }^{2}$, \\ KANAME HIRAYANAGI ${ }^{4}$, TSUKASA SUZUKI ${ }^{2}$, HIROMASA HASEGAWA ${ }^{5}$, TAKANAGA OCHIAI ${ }^{5}$, \\ NOBUHISA YAMAGUCHI ${ }^{4}$, MITSUGU KOCHI ${ }^{1}$, MAKOTO KIMURA $^{2}$ and MARIKO ESUMI ${ }^{3}$ \\ Departments of ${ }^{1}$ Digestive Surgery, ${ }^{2}$ Cancer Genetics, ${ }^{3}$ Pathology and ${ }^{4}$ Hygiene, Nihon University School of Medicine, \\ Tokyo 173-8610; ${ }^{5}$ Department of Oral Pathology, Matsumoto Dental University, Nagano 399-0781, Japan
}

Received December 31, 2010; Accepted March 16, 2011

DOI: $10.3892 /$ etm.2011.236

\begin{abstract}
Recurrence after liver resection for hepatocellular carcinoma (HCC) is a major clinical problem, and prognostic markers for recurrence are urgently required. For 309 HCC cases, segmented linear regression analysis with two segments was performed, and the interval for the early and late recurrence groups was partitioned at the crosspoint (676 days). We investigated whether gene expression in non-tumorous tissues of remnant liver from 39 hepatitis $\mathrm{C}$ virus-positive HCC cases may be associated with early recurrence of this disease. By microarray analysis, 21 genes were identified as candidate recurrence-associated genes. Further gene expression analysis was performed, and the localization and expression of the gene products of these candidate genes were immunohistochemically evaluated. Low expression of the GBP1 gene and high expression of the TSC22D3 gene were significantly (both $\mathrm{P}=0.04$ ) associated with the risk of early recurrence. Through backward step-wise multivariate logistic regression analysis for the 21 candidate genes, high expression of $G B P I$ reduced [odds ratio $(\mathrm{OR})=0.20 ; 95 \%$ confidence interval $(\mathrm{CI})$ 0.06-0.73, $\mathrm{P}=0.02]$ and high expression of TSC22D3 increased the risk of early recurrence $(\mathrm{OR}=19.6 ; 95 \%$ CI 1.14-337.2; $\mathrm{P}=0.04)$. Immunohistochemical analysis revealed that hepatocytes showed strong membranous expression for $G B P 1$ in the late recurrence group, but weak membranous expression for $G B P 1$ in the early recurrence group. TSC22D3 was frequently expressed in lymphocytes and in a few hepatocytes in tissues of the early recurrence group. Our observations suggest that the combination of the high expression of the TSC22D3 gene and low expression of the GBPl gene in the non-tumorous
\end{abstract}

Correspondence to: Dr Mariko Esumi, Department of Pathology, Nihon University School of Medicine, 30-1 Ohyaguchikami-cho, Itabashi-ku, Tokyo 173-8610, Japan

E-mail: mesumi@med.nihon-u.ac.jp

Key words: hepatocellular carcinoma, hepatitis C virus, microarray, early recurrence, non-tumorous tissue tissue of the remnant liver is significantly associated with early recurrence after surgical resection of HCC.

\section{Introduction}

Hepatocellular carcinoma (HCC) is one of the most common human malignancies in the world. Chronic infection with the hepatitis $\mathrm{B}$ or $\mathrm{C}$ virus (HBV or $\mathrm{HCV}$ ) is a major risk factor for HCC. In Japan, approximately $85 \%$ of HCC cases are caused by $\mathrm{HBV}$ and HCV infections (1). In particular, HCV-positive HCC accounts for $72 \%$ of $\mathrm{HCC}$ cases. Although liver resection has been established as a curative treatment for HCC, recurrence is observed with a high frequency after resection. HCC patients suffer relapses in 25,50 and $80 \%$ of cases within 1,2 and 5 years after liver resection, respectively $(2,3)$. Recurrence after liver resection for HCC occurs mostly in the remnant liver. Vascular invasion, tumor size, multiple tumors, cirrhosis, TNM classification, serum $\alpha$-fetoprotein (AFP) level and serum bilirubin level have been established as risk factors for HCC recurrence, $(3,4)$. However, the molecular pathogenesis of $\mathrm{HCC}$ recurrence in the non-tumorous tissues of remnant livers is controversial.

The recent introduction of DNA microarray technology has opened a new road in medical science and has demonstrated the critical molecular and biological characteristics of HCC (5). Comprehensive analyses of the gene-expression profiles of HCC have been performed taking into account various clinical characteristics, such as the differences between tumor and non-tumorous tissues (6), causative hepatitis virus types $(6,7)$, the presence and absence of portal invasion (6) and histological differentiation grades (6), and have revealed a group of significant genes. Moreover, genetic predisposition towards recurrence is thought to be harbored in the tumor environment, such as in a non-tumorous liver with chronic hepatitis or liver cirrhosis. When liver cancer is surgically resected, recurrence occurs in the remaining remnant liver or extrahepatic lesion. In particular, recurrence after liver resection for HCC occurs most frequently in the remnant liver. In the present study, we focused on Japanese HCC patients, the majority of whom presented with HCV-positive HCC. Gene expression was assessed in the non-tumorous tissues of the remnant liver in HCC patients who had an early recurrence compared to that in patients who had late recurrence. 


\section{Materials and methods}

Patients and tissue samples. From March 1995 to December 2006, a total of 309 patients with UICC TNM classification (8) stages I and II HCV-positive HCC underwent liver resection at Nihon University Hospital, Japan. Surgical samples were obtained from 39 of 222 patients who had only a solitary tumor, and in whom no secondary cancerous mass was clinically observed in the remnant liver and no extrahepatic lesions were noted during the initial surgery. Non-tumorous tissues were collected from the negative resected margin, which was pathologically confirmed to be a non-cancerous region. Samples were stored frozen at $-80^{\circ} \mathrm{C}$ immediately after separation. Informed consent was obtained from all of the patients in advance. Recurrence was determined every 2-6 months during the follow-up period.

RNA preparation and oligonucleotide microarray. Total RNA was extracted from the frozen tissues using TRIzol reagent (Invitrogen Corp., Carlsbad, CA, USA), following the manufacturer's protocol. The integrity of the RNA obtained was assessed using an Agilent 2100 BioAnalyzer (Agilent Technologies, Palo Alto, CA, USA). Two groups, non-tumorous cRNA from early recurrence $(<2$ years $)$ cases and that from late recurrence ( $>4$ years) cases, were analyzed. Total RNA $(3.3 \mu \mathrm{g})$ from 3 cases were pooled and used for the synthesis of biotinylated cRNA from each group. Complementary DNA synthesis and in vitro transcription from the cDNA to biotinylated cRNA were performed following the Expression Analysis Technical Manual from Affymetrix Inc. (Santa Clara, CA, USA). A GeneChip Human Genome U133A oligonucleotide microarray (Affymetrix Inc.) included 22,283 human genes. In the present study, each group of subjects was represented by a single microarray chip to screen candidate genes differentially expressed between the groups.

Following the manufacturer's instructions, the microarray chips were subjected to pre-hybridization and hybridization with the biotinylated cRNA. They were then washed and stained. The fluorescence signal on the microarray chips was detected using the Gene Array Scanner (Hewlett-Packard, Palo Alto, CA, USA) and analyzed using GeneSpring version 5.0.3 software (Silicon Genetics, Redwood City, CA, USA). The whole microarray data set of each GeneChip was first normalized to the median of the gene expression intensity values among the genes. The expression signal intensity of each gene was then subjected to further normalization to the median value among the GeneChips. The genes were further screened with the criterion that at least one GeneChip detection flag was present ('P'). These pre-analysis data-processing procedures identified 7,444 genes for expression analysis, following the Data Analysis Fundamentals Manual from Affymetrix Inc. The only criterion modified was the inclusion of signal fold changes for genes that were up-regulated by at least 2.5 or down-regulated by at least -2.5 in the early recurrence non-cancerous tissues compared to the late recurrence non-cancerous tissues.

Real-time RT-PCR. To assess the risk of recurrence using the non-cancerous tissue, RNA was isolated with TRIzol reagent according to the manufacturer's instructions, and the RNA was treated with RNase-free DNase before cDNA synthesis. First-strand cDNA was synthesized using a SuperScript ${ }^{\mathrm{TM}}$ First-Strand kit (Invitrogen Corp.). Real-time RT-PCR (Thermal Cycler Dice ${ }^{\circledR}$ Real Time System TP800; Takara Bio Inc., Shiga, Japan) was carried out using a SYBR ${ }^{\circledR}$ Premix Ex Taq ${ }^{\mathrm{TM}}$ kit or SYBR ${ }^{\circledR}$ Premix Ex Taq ${ }^{\mathrm{TM}}$ II (Takara Bio Inc.). To perform an optimal-condition real-time PCR for each gene, the optimum amount of cDNA was determined and used for each gene. The 25- $\mu$ l two-step RT-PCR mixture consisted of $12.5 \mu \mathrm{l}$ of SYBR Premix Ex Taq, $0.5 \mu \mathrm{l}$ each of a forward and reverse primer, $10.5 \mu \mathrm{l}$ of RNase-free water and $1 \mu \mathrm{l}$ of template cDNA for the PSMB8, RALGDS, APOL3, GBP1, RPS14, CXCL9, DKFZp564F212, CYP1B1, TNFSF10, NROB2, AKR1B10, MAFB, BF530535, MRPL24, TSC22D3, $Q P R T, V N N 1, F M O 5, D C N$ and GAPDH genes, respectively. The 25- $\mu 1$ two-step RT-PCR mixture consisted of $12.5 \mu 1$ of SYBR Premix Ex Taq II, $1.0 \mu$ l each of a forward and reverse primer, $9.5 \mu \mathrm{l}$ of RNase-free water and $1 \mu \mathrm{l}$ of template cDNA for the $A L B$ and $I R S 2$ genes. The real-time cycler conditions consisted of $95^{\circ} \mathrm{C}$ for $10 \mathrm{sec}$ followed by 40 cycles at $95^{\circ} \mathrm{C}$ for $5 \mathrm{sec}$ and $60^{\circ} \mathrm{C}$ for $30 \mathrm{sec}$. The relative quantity for these gene expression levels was normalized to GAPDH expression.

Each cDNA sample was subjected to triplicate RT-PCR reactions, and the results were processed by absolute quantitative analysis based on a standard curve determined by serial 5 -fold dilutions of an appropriate cDNA. Multiple RQ software, ver. 1.00 (Takara Bio Inc.) was used for data analysis. The primer sequences of the internal standard and target genes are shown in Table I.

Immunohistochemistry. To confirm the localization of early recurrence-related gene products in the non-tumorous liver, $4-\mu \mathrm{m}$ sections were routinely prepared from archived paraffinembedded blocks of two selected cases that clearly showed low or high mRNA expression by RT-PCR. Deparaffinized sections were autoclaved in $0.01 \mathrm{M}$ sodium citrate buffer (pH 6.0) at $121^{\circ} \mathrm{C}$ for $15 \mathrm{~min}$. After routine pre-treatments, sections were incubated at $4^{\circ} \mathrm{C}$ overnight with an anti-GBP1 monoclonal antibody (clone 4D10; Abnova, Taipei, Taiwan) at a 1:300 dilution and anti-TSC22D3 (TSC22 domain family, member 3) antibody (clone 3A5; Abnova) at a 1:300 dilution. Histofine simple stain Max PO (Multi) (Nichirei Co., Tokyo, Japan) was used as the secondary antibody, and was applied for $30 \mathrm{~min}$ at room temperature. After visualization with Vector ${ }^{\circledR}$ SG substrate (Vector Laboratories, Burlingame, CA, USA) the sections were counterstained with nuclear fast red. A negative control slide without primary antibody was included in each staining. Nuclei positive for TSC22D3 staining were counted in ten high-power fields.

Classification of early and late recurrence groups. The disease-free survival rate was calculated using the KaplanMeier method. To distinguish the early from the late recurrence group, segmented linear regression analysis with two segments was used to determine a crosspoint of threshold values above and below which it was possible to divide the two groups according to the distribution of the interval of recurrence for either the early or the late recurrence group (Fig. 1). The $\mathrm{y}_{1}=-0.097 \mathrm{x}+98.84$ line for an interval of $\mathrm{x} \leq 676$ days 
Table I. Recurrence-related candidate genes tested by real-time RT-PCR.

Genes

Primer sequence

Forward (5'-3')

Reverse (5'-3')

$\begin{array}{ll}\text { Early recurrence-related } & \\ A L B & \text { CAAAGCATGGGCAGTAGCTC } \\ N R O B 2 & \text { TCTTCAACCCCGATGTGCCA } \\ \text { AKR } 1 B 10 & \text { CTTGGAAGTCTCCTCTTGGC } \\ M A F B & \text { TCTGGGCCTGCGCTAATTG } \\ B F 530535 & \text { AGTGGGATGGATCAGCTGTGAA } \\ M R P L 24 & \text { AAGGAAGGTTTCGAGCGTTT } \\ T S C 22 D 3 & \text { AACAGGCCATGGATCTGGTG } \\ Q P R T & \text { CTGACTTCGCTCTGAAGGTGGA } \\ V N N 1 & \text { GCTGGAACTTCAACAGGGAC } \\ I R S 2 & \text { GCATTCCAGCCCCTATGTTA } \\ F M O 5^{1} & \text { ACACAGAGCTCTGAGTCAGC } \\ F M O 5^{2} & \text { GAAAGGACTGATGACATCG } \\ D C N & \text { CCTCAAGGTCTTCCTCCTTC }\end{array}$

Late recurrence-related

PSMB8
RALGDS
APOL3
GBP1
RPS14
CXCL9
DKFZP564F212
CYP1B1
TNFSF10

AGACTGTCAGTACTGGGAGC

TGCCGCTCTACAACCAGCAG

AATTGCCCAGGGATGAGGCA

AGAGGACCCTCGCTCTTAAACTTC

GACGTGCAGAAATGGCACCT

CCTGCATCAGCACCAACCAA

CCTGGGCAAGTGAGGTCTTC

CCTCTTCACCAGGTATCCTG

GCTGAAGCAGATGCAGGACA

\author{
CAAGCAGATCTCCATGGCAG \\ AGGCTGGTCGGAATGGACTT \\ ATGAACAGGTCCTCCCGCTT \\ TTGGTTCAGTGCAGTGTCTGCTTAC \\ TGGTGAGGCGACCATCAATTAG \\ GAGATGGGTTCCACAACCAC \\ AGGACTGGAACTTCTCCAGC \\ CACAGCCACACTCGGGAACT \\ CTGAGGATCACTGGTATCGC \\ AGTGTCGAGGGAGCAGAAAA \\ TCCAGGTTAGGAGGGAAGAC \\ TGAAATACTCCAGGACCTGG \\ CACCAGGTACTCTGGTAAGC
}

GTCCAGGACCCTTCTTATCC
GAATCTGCAGCAGCTCATAGTCCTC
TGGACTCCTGGATCTTCCTC
TTATGGTACATGCCTTTCGTCGTCT
CAGTCACACGGCAGATGGTT
TGGCTGACCTGTTTCTCCCA
TCTCTGGCAGGTTGTTCCTGA
CCACAGTGTCCTTGGGAATG
CTAACGAGCTGACGGAGTTG

Internal control gene

GAPDH

GCACCGTCAAGGCTGAGAAC

TGGTGAAGACGCCAGTGGA

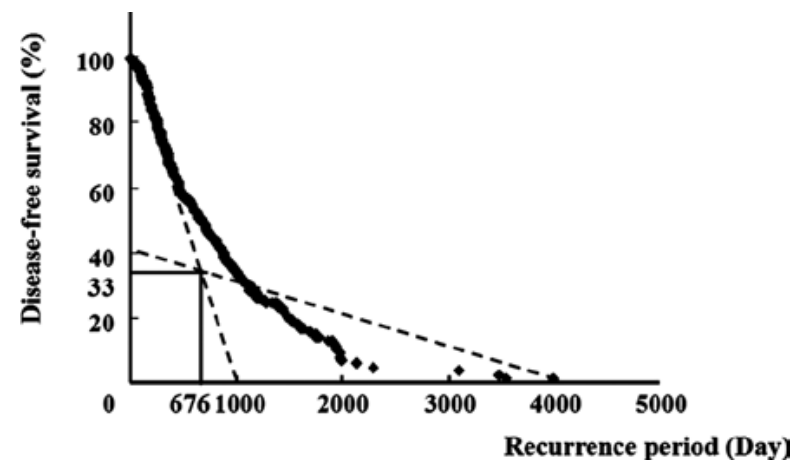

Figure 1 . The crosspoint distinguishing early and late recurrence after liver resection of solitary HCV-positive HCC in stage I/II. The disease-free survival rate was calculated using the Kaplan-Meier method. To distinguish the early from the late recurrence group, segmented linear regression analysis was used. The solid straight line $y_{1}=-0.097 x+98.84$ and the dotted straight line $y_{2}=-0.012 x+41.16$ intersected at 676 days. The early recurrence group was defined as having an interval of recurrence less than the crosspoint, and the late recurrence group, greater than the crosspoint (676 days).

(crosspoint), and the $y_{2}=-0.012 x+41.16$ line for an interval of $x>676$ days (crosspoint) are shown. The goodness of fit was estimated by $R^{2} \cdot R^{2}$ values for the intervals of $x \leq 676$ days and $>676$ days were 0.98 and 0.81 , respectively, suggesting that each group fit a distinct linear regression separated at a crosspoint of 676 days. Thus, the early recurrence group was defined as having an interval of recurrence less than the crosspoint, and the late recurrence group, an interval of recurrence greater than the crosspoint (676 days) (Fig. 1).

Statistical analysis. The disease-free survival rate was calculated from the period begining with the date of surgery until the date of recurrence. Differences in gene expression between the two groups were evaluated using the MannWhitney U-test. The Chi-square and Fisher's exact probability tests were used for discrete variables, and the Student's t-test for continuous variables. A step-wise discriminant analysis was applied to the expression levels of 21 genes isolated from microarray analysis, and a gene combination suitable for distinguishing between the early and late recurrence groups was extracted. The best combination of genes associated with recurrence risk within 2 years after resection was also determined by a backward step-wise multivariate logistic regression analysis using gene expression levels from the non-tumorous livers. Akaike's information criterion (AIC) was used to evaluate the goodness-of-fit of a logistic regression model in each step. Statistical analysis was performed 
using SPSS, version 15.0 (SPSS Inc., Chicago, IL, USA). A P-value of $<0.05$ was considered to indicate statistical significance.

\section{Results}

Comparison of the clinicopathological features between the early and late recurrence groups. Using the Kaplan-Meier method, 676 days was selected as the crosspoint for distinguishing between the early and late recurrence groups. As shown in Fig. 1, the early recurrence group was defined as patients having disease recurrence before 676 days. Among the 39 cases, early recurrence occurred in 22 cases and late recurrence in 17 cases. Table II shows a comparison of background clinicopathological features between the two groups. No significant differences were found among variables, including gender, age, tumor size, histological grade, background liver, indocyanin green retention rate at $15 \mathrm{~min}$, total bilirubin, albumin, aspartate aminotransferase, alanine aminotransferase, platelets, AFP and vascular invasion.

Recurrence-related genes in the non-tumorous liver. Two pooled samples of cRNA each from three representative cases from the early or late non-tumorous recurrence groups were applied to the oligonucleotide microarray expression analysis of non-tumorous liver mRNA. The data analysis referred to a signal of the control gene BioB for an intrinsically fixed quantity after normalization as a detection limit. Probes that had differences in expression of $\geq 2.5$-fold between the two groups were extracted; finally, only probes having P flags on all of the microarrays with increased expression were selected. In this way, 21 of the 22,283 genes were identified as being differentially expressed between the non-tumorous livers of the early and late recurrence groups. Among these genes, 9 were up-regulated in the late recurrence group and 12 were up-regulated in the early recurrence group (Table I).

Prediction of early recurrence genes. To examine whether the 21 candidate genes were differentially expressed between the early and late HCC recurrence groups, mRNAs from the 21 genes in the non-tumorous tissues of all 39 HCC cases were quantified. Between the early and late recurrence group, 2 genes were recognized to exhibit a difference in expression. Significantly low expression of the GBPI gene and significantly high expression of the TSC22D3 gene were noted in the early recurrence group (Table III). No significant difference in expression was noted for the other genes.

The best combination of genes associated with recurrence risk within 2 years after resection was also determined by a backward step-wise multivariate logistic regression analysis using gene expression levels from the non-tumorous livers. The expression of GBP1 and TSC22D3 was associated with the rate of recurrence. The GBPI gene was identified as being associated with a reduced risk of early recurrence [odds ratio $(\mathrm{OR})=0.20]$, while the $T S C 22 D 3$ gene was identified as being associated with an increased risk of early recurrence $(\mathrm{OR}=19.6)$. A 95\% confidence interval (CI) is indicated in Table IV. These two genetic combinations were identified as useful parameters for the prediction of recurrence by multivariate logistic regression analysis (Fig. 2).
Table II. Comparison of clinicopathological features between the early and late recurrence groups.

\begin{tabular}{|c|c|c|c|}
\hline Variable & $\begin{array}{l}\text { Early group } \\
\quad(\mathrm{n}=22)\end{array}$ & $\begin{array}{l}\text { Late group } \\
\quad(\mathrm{n}=17)\end{array}$ & P-value \\
\hline Gender & & & 0.70 \\
\hline Male & 17 & 14 & \\
\hline Female & 5 & 3 & \\
\hline Age (years) & & & 0.81 \\
\hline Median & 65 & 66 & \\
\hline Range & $50-78$ & $48-73$ & \\
\hline Tumor size $(\mathrm{cm})$ & & & 0.50 \\
\hline$<5$ & 20 & 17 & \\
\hline$\geq 5$ & 2 & 0 & \\
\hline Histological grade & & & 0.52 \\
\hline W/D & 9 & 5 & \\
\hline $\mathrm{M} / \mathrm{D}, \mathrm{P} / \mathrm{D}$ & 13 & 12 & \\
\hline Background & & & 0.21 \\
\hline Non-LC & 7 & 9 & \\
\hline $\mathrm{LC}$ & 15 & 8 & \\
\hline ICG15R (\%) & & & 0.52 \\
\hline$<10$ & 9 & 5 & \\
\hline$\geq 10$ & 13 & 12 & \\
\hline T.Bil (mg/dl) & & & 0.50 \\
\hline$<1.2$ & 20 & 17 & \\
\hline$\geq 1.2$ & 2 & 0 & \\
\hline $\mathrm{Alb}(\mathrm{g} / \mathrm{dl})$ & & & 1.00 \\
\hline$<3.5$ & 6 & 4 & \\
\hline$\geq 3.5$ & 16 & 13 & \\
\hline AST (U/l) & & & 1.00 \\
\hline$<40$ & 6 & 5 & \\
\hline$\geq 40$ & 16 & 12 & \\
\hline ALT (U/l) & & & 0.21 \\
\hline$<40$ & 7 & 9 & \\
\hline$\geq 40$ & 15 & 8 & \\
\hline $\operatorname{PLT}\left(10^{9} / 1\right)$ & & & 0.73 \\
\hline$<15$ & 16 & 11 & \\
\hline$\geq 15$ & 6 & 6 & \\
\hline AFP (ng/ml) & & & 0.18 \\
\hline$<20$ & 6 & 9 & \\
\hline$\geq 20$ & 16 & 8 & \\
\hline Vascular invasion & & & 1.00 \\
\hline$(+)$ & 8 & 7 & \\
\hline$(-)$ & 14 & 10 & \\
\hline
\end{tabular}

W/D, well-differentiated; M/D, moderately differentiated; P/D, poorly differentiated. Early group, recurrence before 676 days; Late group, recurrence after 677 days. ICG15R, indocyanin green retention rate at $15 \mathrm{~min}$; T.bil, total bilirubin; Alb, albumin; AST, aspartate aminotransferase; ALT, alanine aminotransferase; PLT, platelet; AFP, $\alpha$-fetoprotein.

Localization of recurrence-related gene products. GBP1 immunostaining revealed strong membranous expression in hepatocytes demonstrating a reticular pattern in the late recurrence group, while relatively weak membranous staining was noted in the early recurrence group (Fig. 3A and B). Upon TSC22D3 staining, endothelial cells, some lymphocytes and 
Table III. Genes exhibiting differential expression between the early and late recurrence groups by the Mann-Whitney U-test.

\begin{tabular}{lccc}
\hline Gene & $\begin{array}{c}\text { Early group } \\
\text { median (range) }\end{array}$ & $\begin{array}{c}\text { Late group } \\
\text { median (range) }\end{array}$ & P-value \\
\hline GBP1 & $0.45(0.24-1.48)$ & $1.23(0.49-4.23)$ & 0.04 \\
TSC22D3 & $1.07(0.12-2.38)$ & $0.81(0.17-2.30)$ & 0.04 \\
\hline
\end{tabular}

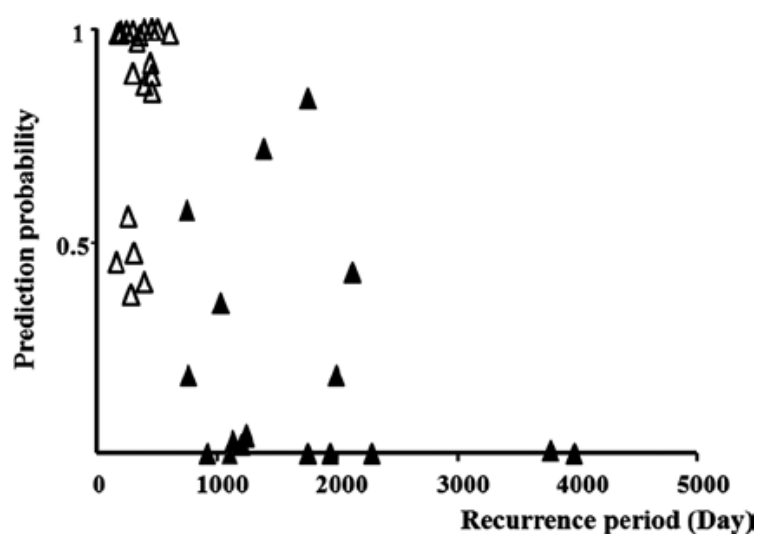

Figure 2. Prediction probability of early and late recurrence. Multivariate logistic regression analysis was applied to identify the independent risk of recurrence. A function for each risk predictor of recurrence was as follows: $\log [\mathrm{p} /(1-\mathrm{p})]=-1.604 \times G B P 1+2.978 \times T S C 22 D 3+0.269$. Black triangle, late recurrence group; white triangle, early recurrence group.

Table IV. Multivariate logistic regression analysis of early recurrence risk.

\begin{tabular}{lclc}
\hline Gene & Odds ratio & $95 \%$ CI & P-value \\
\hline GBP1 & 0.2 & $0.06-0.73$ & 0.02 \\
TSC22D3 & 19.6 & $1.14-337.2$ & 0.04 \\
\hline
\end{tabular}

regenerating bile ductuli exhibited nuclear positivity throughout the sections. The mean values of the positive nuclei were 9.1 and 4.5 in the early and late recurrence groups, respectively. A few nuclei of hepatocytes were positively stained for TSC22D3 in the early recurrence group (Fig. 3C), while there were no positive cells noted in the late recurrence group (Fig. 3D).

\section{Discussion}

Hoshida et al demonstrated the genome-wide expression profiling of formalin-fixed, paraffin-embedded tissues and showed that a reproducible gene expression signature correlating with survival is present in liver tissues adjacent to the tumor in HCC patients (9). Tsuchiya et al reported that various genes in non-tumoral tissues of $\mathrm{HCV}$-associated $\mathrm{HCC}$ cases were associated with late recurrence (10). Here, we identified the early and late recurrence-related genes in non-tumorous tissues from HCV-positive HCC cases. We examined whether the early and late recurrence groups had different patient and tumor characteristics. Although clinically there was no
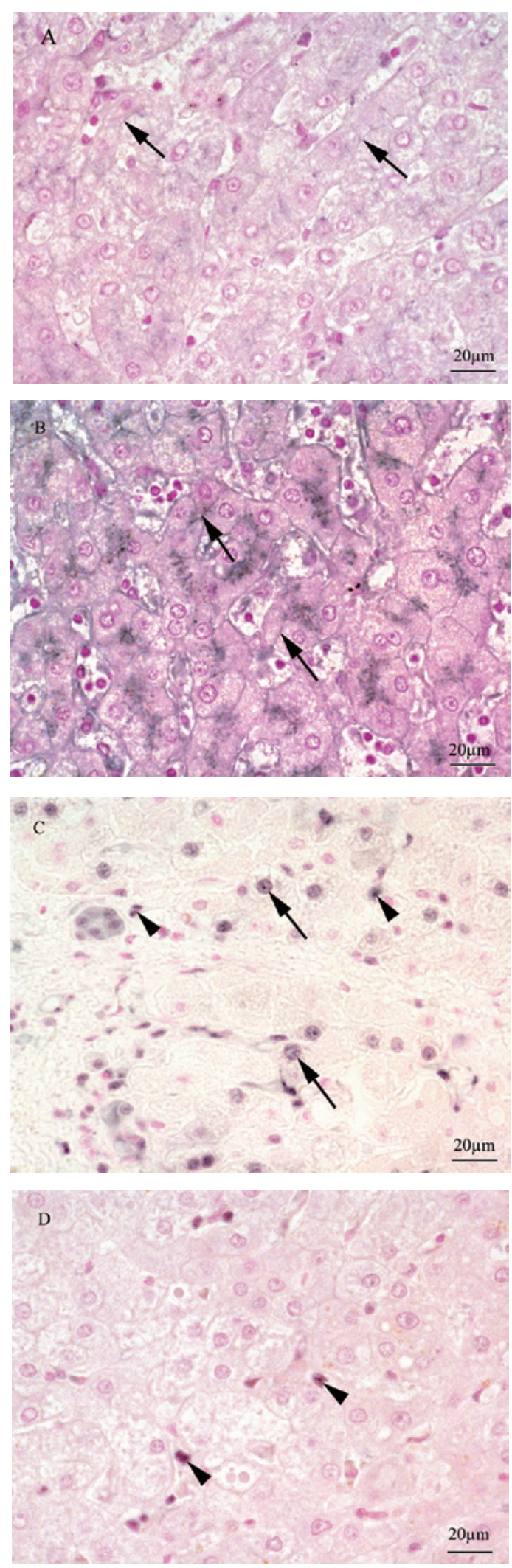

Figure 3. Immunohistochemistry for GBP1 and TSC22D3 in non-tumorous tissues. Some hepatocytes exhibited weak membranous expression (arrows) for GBP1 in the early recurrence group (A), while in the late recurrence group, most hepatocytes demonstrated strong membranous expression (arrows). Some hepatocyes demonstrated cytoplasmic expression for GBP1 (B). Upon TSC22D3 staining, endothelial cells, some lymphocytes and regenerating bile ductuli showed nuclear positivity. In the early recurrence group, positive lymphocytes (arrowheads) were relatively frequent and a few positively stained hepatocytes (arrows) were observed (C). A few positively stained lymphocytes (arrowheads) were also found scattered in the late recurrence group, but no positively stained hepatocytes were observed (D). 
difference when comparing the two groups, we found that the gene expression of two genes could be used to distinguish between them.

Previously, researchers have reported various risk factors for recurrence after liver resection, such as the degree of liver fibrosis (11), hepatitis virus infection type (12), number of tumor nodules $(13,14)$, presence of AFP mRNA in the circulation (15), serum albumin level, ICG-15 level, tumor location (16), presence of venous invasion $(4,17)$ and tumor size $(14)$.

In light of such processes, we aimed to ascertain whether there is a 'bud of recurrence' in the remnant liver, namely in the non-tumorous tissues, and whether this can be defined in postoperative early or late recurrence groups through a gene expression study. Here, non-tumorous tissues were used to investigate whether variations in the expression of $\mathrm{HCC}$ recurrence-related genes affect the recurrence interval. To date, there have been various reports on the gene expression of $\mathrm{HCC}$, but most of the comparisons were carried out between the gene expression of tumor vs. non-tumorous tissues (5-7). Our data clearly showed that the TSC22D3 and GBP1 genes were differentially expressed in the remnant liver tissues of the early and late recurrence groups. Of these, GBP1 exhibited high expression in the late recurrence group, while TSC22D3 demonstrated high expression in the early recurrence group. $G B P 1$, human guanylate binding protein-1, is a member of the large GTPase protein family. Expression of GBPl is induced by inflammatory cytokines (ICs) in endothelial cells (ECs). $G B P 1$ was found to mediate the angiostatic effects of ICs on EC proliferation in vitro (18). Moreover, in vivo GBPl expression was shown to decrease the angiogenic activity of ECs $(18,19)$. Genes associated with HCV-induced HCC, such as GPC 3, HSP70 and TSAP6, have been investigated, while the TSC22D3 and GBP1 genes have not been studied using tumor materials $(20,21)$.

Knockdown of GBPI, IFI-6-16 and IFI-27 by short hairpin RNA was found to result in an increase in $\mathrm{HCV}$ replication (22). Based on our analysis of GBP1 localization, GBP1 appears to be produced by hepatocytes in the non-tumorous tissue of the remnant liver. GBP1 protein expression appeared to be further increased in the late recurrence group than in the early recurrence group. Therefore, increased expression of GBP1 in hepatocytes may suppress HCV replication. GBP1 protein translation may be suppressed in hepatocytes by a degradation pathway, and this pathway may be deregulated in the HCC carcinogenesis process, specifically in early recurrence. The 5-year survival rate was found to be significantly increased in GBP1-positive colorectal cancer patients. GBPI may be a novel biomarker and an active component of the T-helper 1 angiostatic immune reaction in colorectal cancer (23). In this way, GBPl may also control angiostatic effects in the liver.

The expression of TSC22D3 (TSC22 domain family, member 3 ) is stimulated by glucocorticoids and interleukin-10, and appears to play a key role in the anti-inflammatory and immunosuppressive effects of this steroid and chemokine. TSC22D3, also known as glucocorticoid-induced leucine zipper protein (GILZ), is expressed in a variety of mammalian cells. According to a recent report, this gene up-regulated cyclin D1 and phosphorylated retinoblastoma, down-regulated cyclin-dependent kinase inhibitor p21, and promoted entry into the $\mathrm{S}$ phase of the cell cycle in epithelial ovarian cancer (24). HCCs are hypervascular tumors and angiogenesis plays a crucial role in the process of neoplasia (25). In culture, VEGF was found to enhance cyclin D1 expression and HCC cell growth. The restraint of cyclin D1 control was found to decrease angiogenesis (26). In the present study, a few TSC22D3-positive hepatocytes were scattered in the non-tumorous tissue of the early recurrence group, while no positive cells were scattered in the late recurrence group. The physiological role of TSC22D3 has not been clarified, and no previous report on hepatocytes producing TSC22D3 has been published. This seems to suggest that cyclin D1 up-regulated by GILZ and angiogenesis were promoted in the non-tumorous tissues of the early recurrence group.

Here, we investigated recurrence-related genes in the non-tumorous tissues of HCV-positive HCC cases, and demonstrated that $G B P 1$ is associated with a decreased risk of recurrence while TSC22D3 is associated with an increased risk of recurrence. Although there are no reports that these two genes are differentially expressed during liver carcinogenesis, this gene pair appears to be useful as a parameter in the assessment of risk of HCC recurrence.

In conclusion, a combination of high TSC22D3 expression and low $G B P 1$ expression may be considered a risk factor for the early recurrence of HCC after liver resection.

\section{Acknowledgements}

This study was supported by the Nihon University Multidisciplinary Research Grant for 2007; the Academic Frontier Project for 2006 Project for Private Universities; and a matching fund subsidy from MEXT to H.N. The authors thank their colleagues in the Department of Digestive Surgery, and Dr Masako Mitsumata and Dr Norimichi Nemoto of the Department of Pathology, Nihon University School of Medicine. They also thank Ms. Sawako Matsumoto and Dr Hideyo Yasuda, Central Research Institute, Nippon Flour Mills Co., Ltd., for their assistance.

\section{References}

1. Ikai I, Arii S, Okazaki M, et al: Report of the 17th Nationwide follow-up survey of primary liver cancer in Japan. Hepatol Res 37: 676-691, 2007.

2. Makuuchi M, Takayama T, Kubota K, et al: Hepatic resection for hepatocellular carcinoma - Japanese experience. Hepatogastroenterology 45 (Suppl 3): 1267-1274, 1998.

3. Imamura H, Matsuyama Y, Tanaka E, et al: Risk factors contributing to early and late phase intrahepatic recurrence of hepatocellular carcinoma after hepatectomy. J Hepatol 38: 200-207, 2003.

4. Poon RT, Fan ST, Ng IO, Lo CM, Liu CL and Wong J: Different risk factors and prognosis for early and late intrahepatic recurrence after resection of hepatocellular carcinoma. Cancer 89: 500-507, 2000.

5. Honda M, Kaneko S, Kawai H, Sirota Y and Kobayashi K: Differential gene expression between chronic hepatitis B and C hepatic lesion. Gastroenterology 120: 955-966, 2001.

6. Okabe H, Satoh S, Kato T, et al: Genome-wide analysis of gene expression in human hepatocellular carcinomas using cDNA microarray: identification of genes involved in viral carcinogenesis and tumor progression. Cancer Res 61: 2129-2137, 2001.

7. Iizuka $\mathrm{N}$, Oka $\mathrm{M}$, Yamada-Okabe $\mathrm{H}$, et al: Differential gene expression in distinct virologic types of hepatocellular carcinoma: association with liver cirrhosis. Oncogene 22: 30073014,2003 . 
8. Sobin LH and Wittekind C: TNM Classification of Malignant Tumours (UICC). 6th edition. Wiley-Liss, New York, pp81-83, 2002.

9. Hosida Y, Villanueva A, Kobayashi M, et al: Gene expression in fixed tissues and outcome in hepatocellular carcinoma. N Engl J Med 359: 2045-2047, 2008.

10. Tsuchiya M, Parker JS, Kono H, Matsuda M, Fujii H and Rusyn I: Gene expression in nontumoral liver tissue and recurrence-free survival in hepatitis $\mathrm{C}$ virus-positive hepatocellular carcinoma. Mol Cancer 9: 74, 2010.

11. Ko S, Kanehiro H, Hisanaga M, Nagao M, Ikeda $\mathrm{N}$ and Nakajima Y: Liver fibrosis increases the risk of intrahepatic recurrence after hepatectomy for hepatocellular carcinoma. Br J Surg 89: 57-62, 2002.

12. Wakai T, Shirai Y, Yokoyama N, Nagakura S and Hatakeyama K Hepatitis virus status affects the pattern of intrahepatic recurrence after resection for hepatocellular carcinoma. Eur J Surg Oncol 29: 266-271, 2003.

13. Shah SA, Cleary SP, Wei AC, et al: Recurrence after liver resection for hepatocellular carcinoma: risk factors, treatment, and outcomes. Surgery 141: 330-339, 2007.

14. Regimbeau JM, Abdalla EK, Vauthey JN, et al: Risk factors for early death due to recurrence after liver resection for hepatocellular carcinoma: results of a multicenter study. J Surg Oncol 85: 36-41, 2004

15. Ijichi M, Takayama T, Matsumura M, Shiratori Y, Omata M and Makuuchi M: $\alpha$-fetoprotein mRNA in the circulation as a predictor of postsurgical recurrence of hepatocellular carcinoma: a prospective study. Hepatology 35: 853-860, 2002.

16. Chen JY, Chau GY, Lui WY, Tsay SH, King KL and Wu CW: Clinicopathologic features and factors related to survival of patients with small hepatocellular carcinoma after hepatic resection. World J Surg 27: 294-298, 2003.

17. Shirabe K, Kanematsu T, Matsumata T, Adachi E, Akazawa K and Sugimachi K: Factors linked to early recurrence of smal hepatocellular carcinoma after hepatectomy: univariate and multivariate analyses. Hepatology 14: 802-805, 1991.
18. Guenzi E, Töpolt K, Lubeseder-Martellato C, et al: The guanylate binding protein-1 GTPase controls the invasive and angiogenic capability of endothelial cells through inhibition of MMP-1 expression. EMBO J 15: 3772-3782, 2003.

19. Guenzi E, Töpolt K, Cornali E, et al: The helical domain of GBP-1 mediates the inhibition of endothelial cell proliferation by inflammatory cytokines. EMBO J 20: 5568-5577, 2001.

20. Llovet JM, Chen Y, Wurmbach E, et al: A molecular signature to discriminate dysplastic nodules from early hepatocellular carcinoma in HCV cirrhosis. Gastroenterology 131: 1758-1767, 2006.

21. Smith MW, Yue ZN, Geiss GK, et al: Identification of novel tumor markers in hepatitis $\mathrm{C}$ virus-associated hepatocellular carcinoma. Cancer Res 63: 859-864, 2003

22. Itsui Y, Sakamoto N, Kurosaki M, et al: Expressional screening of interferon-stimulated genes for antiviral activity against hepatitis C virus replication. J Viral Hepat 13: 690-700, 2006.

23. Naschberger E, Croner RS, Merkel S, et al: Angiostatic immune reaction in colorectal carcinoma: impact on survival and perspectives for antiangiogenic therapy. Int J Cancer 123: 2120-2129, 2008.

24. Redjimi N, Gaudin F, Touboul C, et al: Identification of glucocorticoid-induced leucine zipper as a key regulator of tumor cell proliferation in epithelial ovarian cancer. Mol Cancer 8: 83, 2009.

25. Wu XZ, Xie GR and Chen D: Hypoxia and hepatocellular carcinoma: the therapeutic target for hepatocellular carcinoma. J Gastroen Hepatol 22: 1178-1182, 2007.

26. Yasui M, Yamamoto H, Ngan CY, et al: Antisense to cyclin D1 inhibits vascular endothelial growth factor - stimulated growth of vascular endothelial cells: implication of tumor vascularization. Clin Cancer Res 12: 4720-4729, 2006. 
